\title{
The Impact of Ramadan on Online Learners Behaviour: What Can We Learn from Tracking Learners' Data?
}

\author{
Wad Ghaban \\ School of computer \\ science \\ University of Birmingham \\ B15 2TT \\ whg360@alumni.bham.ac.uk
}

\author{
Robert Hendley \\ School of computer \\ science \\ University of Birmingham \\ B15 2TT \\ r.j.hendley@cs.bham.ac.uk
}

\author{
Rowanne Fleck \\ School of computer \\ science \\ University of Birmingham \\ B15 2TT \\ R.fleck@cs.bham.ac.uk
}

\begin{abstract}
The flexibility of online courses means that people are able to fit them around other commitments, often studying during holiday times. However, there is limited research examining the behaviour of online learners during holidays and special events. It is important to understand this in order to know when learners will be active and therefore when they might be contactable or require online support. We conducted this study to examine the changes in behaviour of online learners during Ramadan, a period when Muslims are required to fast between sunrise and sunset. This change in mealtimes affects Muslims' wake-sleep cycles and work patterns as well as all other aspects of their lives. The results can be used to infer an understanding of their lifestyle during this period.

The insights that this provides are important in the context of online learning but also many other application domains where users' availability and openness to interaction are important.
\end{abstract}

Tracking users, Online learning, Behaviour, Holiday, Ramadan, Activity

\section{INTRODUCTION}

Online learning is popular because of its flexibility learners can learn at the time and location of their choice. This flexibility attracts many users during the holidays. However, schedules and sleep patterns can be different when students are not in school/college or at work. These changes in behaviour should be accounted for when designing online courses. For this kind of courses, it is important to know when learners are available and able to interact with live lectures (Shelton et al. 2017). There is little research exploring how learners interact with online courses during special occasions such as holidays. This may be because of the difficulty of reaching participants during these times (Harrison et al. 2015); (Vlajic et al. 2018). One of the special holidays that has been widely accepted as a subject of interest is the holy month of Ramadan. Ramadan is a religious month for Muslims. All healthy, adult Muslims are required to refrain from eating any food, drinking any liquid or taking any oral medication during daylight hours (Ramadan 2002). Most of the research studies addressing this period have investigated the effect of fasting on Muslims' body fat and their health. A few studies have tried to examine the behaviour of Muslims during Ramadan (Roky et al. 2001); (Reilly and Waterhouse 2007).
However, the results from these studies may not be reliable due to issues related to their methodology, particularly the small number of Muslims involved in the studies. Further, these studies were based on self-report questionnaires.

In the light of this gap in the research, we ran a study using a more objective approach by tracking the activity of 564 learners to observe their usage patterns when using an online learning website.

The results from this study, and similar studies, show that during Ramadan, the optimal time to contact learners is during the night. These results provide important data that can be used to build hypotheses about the behaviour of users of online courses. A central goal of this research is to inform the design of personalised models that can be used to drive adaption to match both individual users' characteristics and variations in behaviour over time, especially during special events.

This research is important for applications such as online learning, but also for other domains where understanding users' behaviour and when to interact with users is crucial. For instance, adaptive notification systems need to build models to predict opportune moments for interruptions. While these models often make allowances for different behaviour during weekends, they should also be able to cope with special periods such as religious festivals, celebrations, illness and so on. 


\section{BACKGROUND}

Learning is a continuous and active process (Ally 2004). However, researchers have found that over holidays, students lose some of what they have learned in school (Shelton et al. 2017). To mitigate this drop, many families encourage their children to take advantage of online courses during the holidays (Shinwell and Defeyter 2017). To make these online courses more effective, it is important to understand how user behaviour changes and when students are available. Knowing when users are accessing the platform can help the designers to serve users better and provide them with a better user experience.

One popular holiday in Muslim community is Ramadan in which the altered cycle of sleeping and waking is changed (Roky et al. 2001). Several studies have sought to document changes in sleepwake patterns during Ramadan. For example, Wilson et al. (2009) examined 20 male soccer players to explore whether there was any significant difference between their sleep-wake patterns during Ramadan compared to normal times. The results showed that their sleeping and waking times during Ramadan differed significantly from their sleeping and waking times in other months. These results support the finding from (Roky et al. 2001). In this study, they asked 8 Muslims to report their sleep time and sleep duration before, during and after Ramadan. They found that sleep time is delayed and sleep duration is decreased during Ramadan.

Most of the previous studies have been based on self-reported information that was obtained by asking participants about their behaviour during Ramadan (Mehmood et al. 2015); (Yıldırım-Yenier et al. 2016). They also have quite small numbers of participants. Further, to the best of our knowledge, no study has tried to understand how individuals learn or study during Ramadan. This is partly because Ramadan is a public holiday in most Muslim countries. However, in some countries, many people work during Ramadan. Even on working days, however, Bahammam (2006) shows that these Muslims follow a specific pattern by staying up all night and resuming their sleep after finishing their work.

To address this lack of understanding, we designed the current study. The aim is to analyse the behaviour of online learners during Ramadan by monitoring their use of an online learning website and comparing this with usage patterns outside of Ramadan.

\subsection{Method}

This study aimed to develop an understanding of the ways in which online learners interact with a learning website during Ramadan, and what subtle changes occur in their behaviours.
For this purpose, we asked 564 Muslims learners in Saudi Arabia to register in an online learning platform. After registration, participants were divided in two groups: one group, 194 Muslims learners (91 boys, 103 girls), used the online learning website before Ramadan (4th-29th September 2018), and the second group, 370 Muslims learners (169 boys and 201 girls), used the same online learning website during Ramadan (10th May-4th June 2019). During the designated time period, learners were free to use the website at any time and in any place. Further, we told the learners they were free to drop out of the study whenever they wanted. The platform supported with chat feature, which participants are able to talk to their friends by clicking on a button called 'talk to a friend'.

In the beginning of our study, we measured the number of learners who interacted and engaged with the online learning website during Ramadan and non-Ramadan days. We calculated the number of active learners as each time the learner accessed the website; if a learner accessed the website twice in a single hour, the system registered the activity two separate times.

In 2019, Ramadan began on Monday, May 6th. and continued until Monday, June 3rd.. During this time, Muslims are expected to fast from sunrise until sunset. The time of day at which the sun rises and sets varies according to geographical location and changes throughout the period. We collected all the data in this study from participants in northwest Saudi Arabia. The times at which the sun rises and sets change few minutes between the beginning and the end of Ramadan.

The system began recording users' activities during Ramadan on May 10, 2019, which was the fifth day of Ramadan. Because we were only concerned about the number of users who were active during this time, the system only registered the number of users accessing the website. We used this dataset to determine whether there was a common pattern in user activity. We then compared this data with data from the non-Ramadan days.

\section{RESULTS}

Figure 1 shows the average percentage of learners who were active online on both the Ramadan and non-Ramadan days. During Ramadan, learners began their activity after breaking their fast (after sunset). Further, these learners stayed awake all night and into the early hours of the morning. This contrasts with the activity of the learners on normal (non-Ramadan) days, when they were more active and interacted with the Internet during the day, especially after school.

Because of the interesting change in the learners' behaviours during Ramadan, we examined the data we obtained during Ramadan in more detail. We monitored the number of active daily learners from May 10 to June 4, the last day of Ramadan. We 
notice some dips in the usage of the online learning system on specific days, such as the 17th, 24th and 31 st of Ramadan. We found that all of these dates were Fridays.

Further, the number of active learners rises until the middle of Ramadan before dropping at the end of Ramadan.

After plotting the total activity of all the learners, we examined whether there is a variation between the activity of the boys and the girls (figures 2,3,4 and 5.)

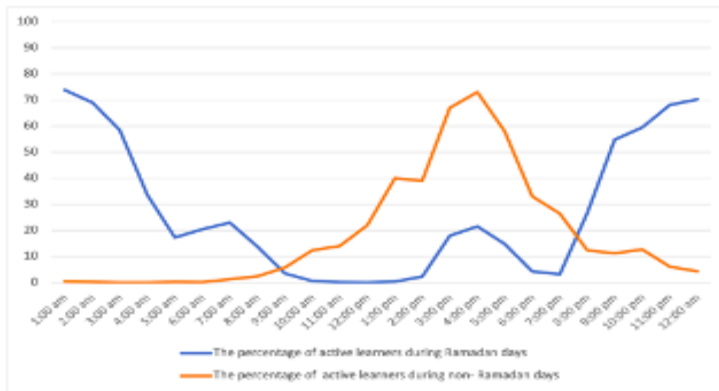

Figure 1: The variation in the activity of the users during both Ramadan and non-Ramadan days

\section{DISCUSSION}

The results from our study supported the findings from related research studies (Roky et al. 2001); (Bahammam2006); (Reilly and Waterhouse 2007). Most Muslims became active during the night, which is considered an interesting phenomenon and is not part of any cultural tradition. Most Muslims interacted with the online learning platform during the night which is the standard time to eat and drink. In addition, this study took place in Saudi Arabia, where this Ramadan occurred in the summer season. The accompanying high temperatures may have had an effect on user behaviour, as users may have preferred to stay home and sleep rather than be awake and active in the hot weather during the day. This became clear when we analysed the messages exchanged between learners. For example, one learner typed, 'Why should I wake up early, there is nothing to do and it is hot!' Another learner wrote that he or she slept during the day because most places, such as shops and malls, were closed. As one learner wrote, 'If I wake up earlier, what I should do. All [the] malls and shopping centres are closed, and of course, restaurants are closed as well.'

Another interesting point to consider is the observed variation in behaviour between boys and girls. The overall numbers of boys and girls who were active on the website were quite similar during each hour over the entire month. However, we noticed some differences in their behaviour. For example, in figures $2,3,4$ and 5 . we see that girls were active earlier in the day than boys, and that boys stayed awake later. Further, the number of active learners changed over Ramdan. This number rose by the middle of the Holy Month
Finally, there were again only a few active learners during the days before the end of Ramadan, with a notably smaller numbers of girls. This can be explained by preparations for Eid. In most Muslim countries, it is traditional that women spend time shopping for new clothes, furniture and decorations around this time to celebrate the end of the fast.

Another observation made during this study is the variation in learners' behaviour between different days of the week. For example, Friday is considered a special day of the week, as male Muslims must attend mosques to pray a special prayer in the afternoon called aljuma'a. Accordingly, we noted that more learners were active in the late morning on Fridays.

Figure 6 shows learners' activity on Fridays and compares it to the average learners' activity on other days of the week.

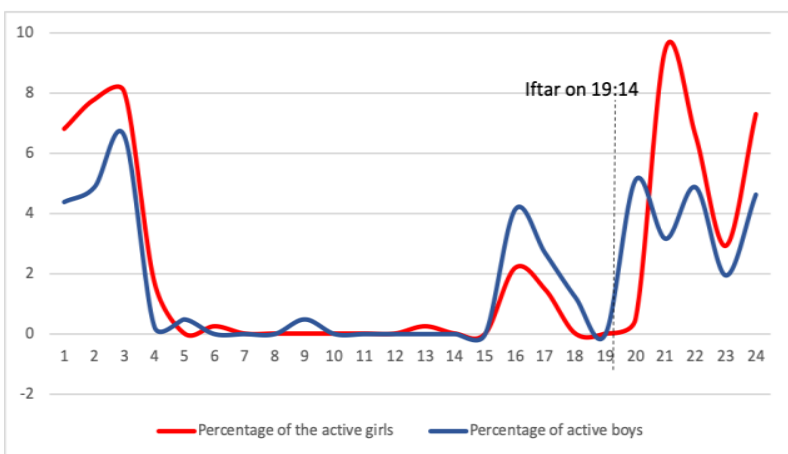

Figure 2: The activity of users on the $11^{\text {th }}$. May



Figure 3: The activity of users on the $18^{\text {th }}$. May

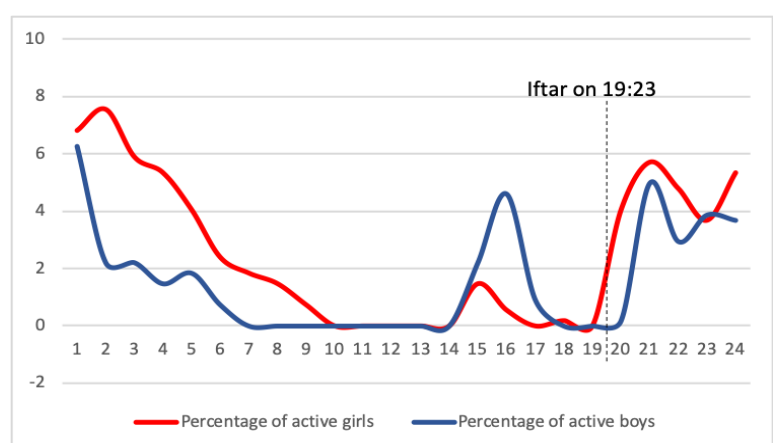

Figure 4: The activity of users on the $25^{\text {th }}$. May 


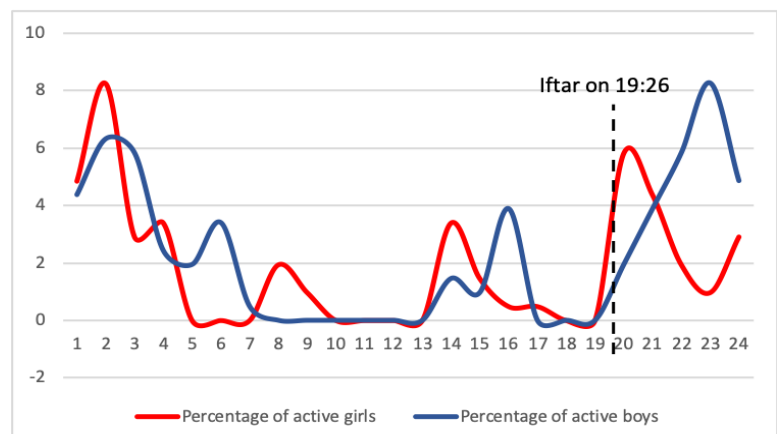

Figure 5: The activity of users on the 1st. June

Our results could help identify the optimal time to contact Muslims during Ramadan, in particular in relation to when to publish online content or for advertising. Finally, the findings could also be compared to the results of other studies to identify similarities and differences between the behaviour of Muslims living in different regions. This data can be used as part of digital ethnography research to develop insights into Internet-user behaviour during Ramadan.

In this research, we collected data from teenage learners who voluntarily visited a learning website, but the results may be generalisable to other Muslims living in the same geographical area (Saudi Arabia). Our results confirmed what what Al-Ghadir and Azmi (2019) suggested. In their research, they tracked the number of tweets from adults during Ramadan.

Of course, this study was undertaken with a very narrow user group: young adults in one geographical area. Further work should be done to explore whether these results can be generalised to other age groups and to other countries. In order to obtain more generalisable data, we may need to repeat the study with predominantly Muslim users in other geographical locations. However, Ramadan occurs only once a year, which makes it difficult to repeat the study multiple times. In addition, we only focused on teenage learners, who do not have as many responsibilities as adults. Generalising the results to other populations would require tracking the activity of adults using online learning courses or other online systems.

\section{CONCLUSION}

The aim of this research was to investigate the behaviour of online learners in Saudi Arabia during Ramadan. Within this work we argue that this change in the observable on-line behaviour can be used to provide insight into the broader changes to users' pattern of living. The results from this study show that there is a variation in the active time of the learners during Ramadan versus non-Ramadan days.

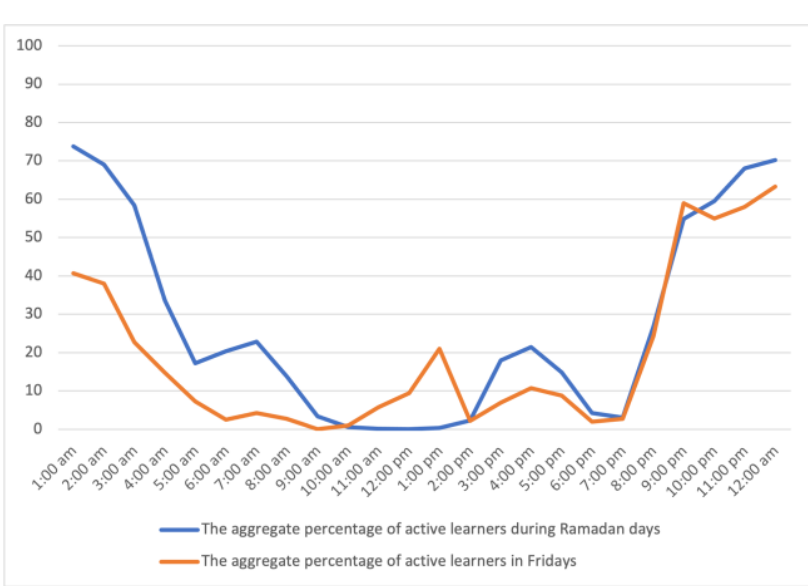

Figure 6: The activity of the users in Fridays compared with the other days

Our results provide an understanding of learners' behaviour during Ramadan. This understanding is important in the context of online learning. For example, it may possible to use the results from these studies to build an adaptive model that presents the learning content based on learners' optimal time. Further, the results also can be used to identify the best time to connect with Muslims during Ramadan.

The insights provided by the analysis of this digital data are important. Although these changes in behaviour have been understood anecdotally, this is the first study to report results based on objective data and with a significant number of research subjects.

This kind of study is important for investigating the behaviour of Muslims during Ramadan, and similar approaches could be used to explore other special events and periods. While many adaptive systems do take account of the differences between weekdays and weekends, there are actually a very large number of exceptional periods; thus building an average model of behaviour that ignores these differences will only have limited success.

There are many applications of our findings to domains in which a model of user availability might be used to determine the most appropriate time to issue notifications or interact with users. For instance, choosing the right time to push a social media notification or to post an advertisement has a large impact on its effectiveness.

The results from the study reveal the difficulty of building a constant model for users during different times and occasions. However, it is important to improve the user experience by building a dynamic model that recognises the time and the user status, then provides customised content based on the provided information. 


\section{REFERENCES}

Al-Ghadir, A. I. and Azmi, A. M. (2019), 'A study of arabic social media users-posting behavior and author's gender prediction', Cognitive Computation 11(1), 71-86.

Ally, M. (2004), 'Foundations of educational theory for online learning', Theory and practice of online learning 2, 15-44.

Bahammam, A. (2006), 'Does ramadan fasting affect sleep?', International journal of clinical practice 60(12), 1631-1637.

Harrison, D., Marshall, P., Bianchi-Berthouze, N. and Bird, J. (2015), Activity tracking: barriers, workarounds and customisation, in 'Proceedings of the 2015 ACM International Joint Conference on Pervasive and Ubiquitous Computing', ACM, pp. 617-621.

Ramadan, J. (2002), 'Does fasting during ramadan alter body composition, blood constituents and physical performance?', Medical Principles and Practice 11(2), 41-46.

Reilly, T. and Waterhouse, J. (2007), 'Altered sleep- wake cycles and food intake: The ramadan model', Physiology \& behavior 90(2-3), 219-228.

Roky, R., Chapotot, F., Hakkou, F., Benchekroun, M. T. and Buguet, A. (2001), 'Sleep during ramadan intermit- tent fasting', Journal of sleep research 10(4), 319-327.

Shelton, B. E., Hung, J.-L. and Lowenthal, P. R. (2017), 'Predicting student success by modeling student interaction in asynchronous online courses', Distance Education 38(1), 59-69.

Shinwell, J. and Defeyter, M. A. (2017), 'investigation of summer learning loss in the ukimplications for holiday club provision', Frontiers in public health 5, 270.

Vlajic, N., El Masri, M., Riva, G. M., Barry, M. and Doran, D. (2018), Online tracking of kids and teens by means of invisible images: Coppa vs. gdpr, in 'Proceedings of the 2nd International Workshop on Multimedia Privacy and Security', ACM, pp. 96103.

Wilson, D., Drust, B. and Reilly, T. (2009), 'Is diurnal lifestyle altered during ramadan in professional muslim athletes?', Biological Rhythm Research 40(5), 385-397. 